Pacific Journal of Mathematics

LIOUVILLE'S THEOREM 


\section{LIOUVILLE'S THEOREM}

\section{RoBert PhILLIPS}

Liouville's theorem states that in Euclidean space of dimension greater than two, every conformal mapping must, by necessity, be an elementary transformation (i.e., a translation, a magnification, an orthogonal transformation, a reflection through reciprocal radii, or a combination of these transformations). This theorem was proven by $R$. Nevanlinna under the additional assumption that the mappings be at least four times differentiable. We show that a modified version of Nevanlinna's proof is still valid when the mappings are assumed to be only twice differentiable. Our methods are those of Nonstandard Analysis as developed by A. Robinson.

We begin with a brief introduction to this subject.

1. Nonstandard Analysis. It is known that there exist proper extensions of the real numbers which possess the same formal properties as the real numbers [Robinson [1]). That is, given a formal mathematical language $L$ in which the algebraic and topological properties of the real numbers $R$ can be expressed, there will exist a proper set-theoretical extension $* R$ of $R$ with the following property: any sentence of $L$ which holds (in the model-theoretic sense) in $R$ will hold in ${ }^{*} R$. Then, since we have assumed that the ordered field axioms which hold in $R$ are expressible in $L,{ }^{*} R$ will be an ordered field; whence, it follows that ${ }^{*} R$ is nonarchimedian. Therefore, there will exist in ${ }^{*} R$ elements $a \neq 0$ that have the property that $|a|<r$ for all positive $r$ in $R$. These elements are called infinitesimal. If $a-b$ is an infinitesimal, we shall write $a \approx b$. If " $0 "$ is regarded as an infinitesimal then it is clear that " $\approx$ " is an additive congruence relation on ${ }^{*} R$. Because $R$ has the formal property " that for each positive number $r$ there will exist a natural number $n$ so that $n>r$ ", ${ }^{*} R$ also has this property. Thus since ${ }^{*} R$ contains infinite numbers (reciprocals of infinitesimals), it follows that ${ }^{*} R$ has infinite natural numbers. That is, embedded in ${ }^{*} R$ is a proper set-theoretical extension of $* N$ of $N$ ( $N$ denotes the natural numbers) which has the same formal properties as $N$. The numbers in ${ }^{*} N-N$ are just the infinite natural numbers.

If $E_{n}$ denotes Euclidean space of dimension $n$, we denote by ${ }^{*} E_{n}$, the natural extension of $E_{n}$ induced by $* R$. That is, elements of ${ }^{*} E_{n}$ are ordered $n$-tuples of elements of $* R$. If $a=\left(a_{1}, \cdots, a_{n}\right)$ and $b=$ $\left(b_{1}, \cdots, b_{n}\right)$ are elements of ${ }^{*} E_{n}$ then $a \approx b$ means 


$$
\sqrt{\left(a_{1}-b_{1}\right)^{2}+\cdots+\left(a_{n}-b_{n}\right)^{2}} \approx 0 \text {. }
$$

2. If $y$ maps an open neighborhood of the point $x$ in $E_{n}$ into $E_{m}$ we say $y$ is differentiable (Frechet differentiable) at $x$ if there exists a bounded linear operator $L$ mapping from $E_{n}$ into $E_{m}$ so that

$$
\lim _{h \rightarrow 0} \frac{y(x+h)-y(x)-L(h)}{|h|}=0 .
$$

We will in this case, denote $L(h)$ by $y^{\prime}(h, x)$. Higher order differentials can be defined as follows: If $h$ is fixed and $f(x)=y^{\prime}(h, x)$ then

$$
\begin{aligned}
& y^{\prime \prime}(k, h, x)=f^{\prime}(k, x) \\
& y^{(3)}(j, k, h, x)=f^{\prime \prime}(j, k, x), \text { etc. }
\end{aligned}
$$

We will sometimes write $y^{\prime} h, y^{\prime \prime} k h, y^{(3)} j k h$, or simple $y^{\prime}, y^{\prime \prime}$ etc., when statements about the differential do not depend on particular values of the arguments. The higher order differentials are symmetric (Nevanlinna [2] p. 85). That is, $y^{\prime \prime} k h=y^{\prime \prime} h k, y^{(3)} j h k=y^{(3)} h j k=y^{(3)} h k j$, etc.

3. We now state the Nonstandard theorems concerning continuity and differentiation which will be needed in this paper:

Theorem 3.1. Let $f$ be a function defined on a set $S$ in $E_{n}$ mapping into $E_{m}$. Then $f$ is uniformly continuous on $S$ if and only if $f(x) \approx f(y)$ whenever $x \approx y$ for any $x$ and $y$ belonging to ${ }^{*} S\left({ }^{*} S\right.$ denotes the extension in $* E_{n}$ of $S$. For instance, if $S$ is the sphere $|x|<r$ then $* S$ includes all points a of $* E_{n}$ which satisfy $|a|<r$. In this case ${ }^{*} S$ will be a proper extension of $S$ ).

For the proof the reader is referred to Robinson [1], p. 111.

THEOREm 3.2. Let $S$ be a closed sphere in $E_{n}$. Suppose $f$ maps $S$ into $E_{m}$ and $f^{\prime}$ exists and is continuous on $S$. Then

$$
\frac{f(x+t h)-f(x)}{t} \approx f^{\prime}(h, x)
$$

holds for each $x$ in ${ }^{*} S$, each $! h$ in $E_{n}$, and for each real $t$ in ${ }^{*} R$ which satisfies $t \approx 0, t \neq 0$.

Proof. It is not difficult to show that the limit

$$
\lim _{t \rightarrow 0} \frac{f(x+t h)-f(x)}{t}=f^{\prime}(h, x)
$$


exists uniformly on $S$. This is because $f^{\prime}$ exists and is uniformly continuous on $S$. But then the result follows based on a theorem similar to Theorem 4.6.1 (Robinson [1], p. 116).

4. We are now in a position to prove the desired result. Let $S$ be an open sphere about the origin in $E_{n}$ and suppose $y$ maps $S$ into $E_{n}$ in a one-to-one manner. Assuming $n>2, y(0)=0$, and that $y^{\prime}, y^{\prime \prime}, y^{(3)}$ and $y^{(4)}$ all exist on $S$ we have:

(4.1) Liouville's Theorem. If $y$ is conformal on $S$, then $y$ is an elementary transformation.

The proof of this theorem is found in Nevanlinna [1]. We sketch this proof in order to make reference to it in the sequel :

If $y$ is conformal, we have

$$
\left|y^{\prime}(h, x)\right|=\lambda(x)|h|
$$

where $\lambda(x)$ is a real function. Setting $\rho(x)=1 / \lambda(x)$, it is not difficult to obtain the equation

$$
\rho^{\prime} k \cdot y^{\prime} h+\rho^{\prime} h \cdot y^{\prime} k+\rho \cdot y^{\prime \prime} h k=0
$$

whenever $h$ and $k$ are orthogonal. Differentiating (4.2) with respect to a vector $j$ orthogonal to $h$ and $k$ we have

$$
\begin{aligned}
& \rho^{\prime \prime} j k \cdot y^{\prime} h+\rho^{\prime} k \cdot y^{\prime \prime} j h+\rho^{\prime \prime} j h \cdot y^{\prime} k+\rho^{\prime} h \cdot y^{\prime \prime} j k \\
& +\rho^{\prime} j \cdot y^{\prime \prime} h k+\rho \cdot y^{\prime \prime \prime} j h k=0 .
\end{aligned}
$$

The sum of the last five terms of (4.3) are symmetric in $j$ and $h$ and hence, the same applies to the first term. We have then,

$$
\rho^{\prime \prime} j k \cdot y^{\prime} h=\rho^{\prime \prime} h k \cdot y^{\prime} j \text {. }
$$

But $y^{\prime} h$ and $y^{\prime} j$ are independent; hence

$$
\rho^{\prime \prime} h k=0
$$

whenever $h$ and $k$ are orthogonal. From this, it follows that for all $h$ and $k$, (see Nevanlinna [1], footnote on p. 7)

$$
\rho^{\prime \prime} h k=q(h \cdot k)(h \cdot k \text { denotes the innerproduct of } h \text { and } k),
$$

$q$ a function of $x$ alone. But differentiating once again with respect to $j, q$ can be shown to be a constant. We then conclude that

$$
\rho(x)=\alpha\left|x-x_{0}\right|^{2}+\beta,
$$

where $\alpha$ and $\beta$ are constants $(\alpha=q / 2)$ and $x_{0}$ a constant vector. The 
fact that $y$ has an inverse, $x=x(y)$, is used to show that either $\alpha$ or $\beta$ is zero. When $\alpha=0$,

$$
\left|y^{\prime}(h, x)\right|=\frac{1}{\beta}|h|
$$

and when $\beta=0$

$$
\left|y^{\prime}(h, x)\right|=\frac{|h|}{\alpha\left|x-x_{0}\right|^{2}} .
$$

In either case, it is not difficult to show that $y$ is an elementary transformation.

Our point is this: the assumption that the third and fourth differentials of $y$ exist is used only to derive equation (4.5) from equation (4.2). We are now going to carry out this derivation without reference to these differentials. The remainder of our proof will be identical to Nevanlinna's and we will not reproduce it here. Hence, we assume that $n>2, y^{\prime}, y^{\prime \prime}$ exist and are continuous on $\bar{S}(\bar{S}$ denotes the closure of $S$ ) and that $y(0)=0$. Under these conditions we prove :

4.6. LIOUVILle's THEOREM. If $y$ is conformal on $S$ then $y$ is an elementary transformation.

Proof. We begin with (4.2)

$$
\rho^{\prime} k \cdot y^{\prime} h+\rho^{\prime} h \cdot y^{\prime} k+\rho \cdot y^{\prime \prime} h k=0
$$

which means that

$$
\left(\rho \cdot y^{\prime} k\right)^{\prime} h=-\rho^{\prime} k \cdot y^{\prime} h
$$

(where $\left(\rho \cdot y^{\prime} k\right)^{\prime} h$ denotes the differential of $\rho \cdot y^{\prime} k$ with respect to $h$ ) holds when $h$ is orthogonal to $k$. Fixing $h$ and $k$ let

$$
F(x)=\rho(x) \cdot y^{\prime}(k, x),
$$

for $x$ in $S$. Then since $F^{\prime}$ exists and is continuous on $\bar{S}$,

$$
F(x+a h)-F(x)=\int_{0}^{1} F^{\prime}(a h, x+t a h) d t
$$

for a real positive $a$. Since $F^{\prime}$ is linear in $a h$ we may divide by $a$ to obtain

$$
\frac{F(x+a h)-F(x)}{a}=\int_{0}^{1} F^{\prime}(h, x+t a h) d t .
$$

If we let $j$ be a fixed vector orthogonal to $h$ and $k$ we then have that 


$$
\begin{aligned}
& \frac{F(x+a h+a j)-F(x+a j)-F(x+a h)+F(x)}{a^{2}} \\
& =\int_{0}^{1} \frac{F^{\prime}(h, x+a j+t a h)-F^{\prime}(h, x+t a h)}{a} d t
\end{aligned}
$$

holds for all $x$ in $S$.

From the definition of ${ }^{*} E_{n}$, it will follow that ${ }^{*} E_{n}$ obeys the same formal properties which hold in $E_{n}$. Thus the fact that (4.9) holds in $E_{n}$ for all positive $a$ in $R$ will imply that (4.9) holds in ${ }^{*} E_{n}$ when $a$ is a positive infinitesimal in ${ }^{*} R$. In this case, it is to be understood that (4.9) holds for all $x$ in ${ }^{*} S$.

Now making use of the uniform continuity of $y^{\prime \prime}$ on $\bar{S}$ (and hence, the uniform continuity of $\rho^{\prime}$ on $\bar{S}$ ) we have by (4.8)

$$
\begin{aligned}
& \frac{F(x+a h+a j)-F(x+a j)-F(x+a h)+F(x)}{a^{2}} \\
& =\frac{\rho(x+a h+a j)-\rho(x+a j)-\rho(x+a h)+\rho(x)}{a^{2}} \cdot y^{\prime}(k, x+a h+a j) \\
& +\frac{\rho(x+a j)-\rho(x)}{a} \cdot \frac{y^{\prime}(k, x+a h+a j)-y^{\prime}(k, x+a j)}{a} \\
& +\frac{\rho(x+a h)-\rho(x)}{a} \cdot \frac{y^{\prime}(k, x+a h+a j)-y^{\prime}(k, x+a h)}{a} \\
& +\rho(x) \cdot \frac{y^{\prime}(k, x+a h+a j)-y^{\prime}(k, x+a h)-y^{\prime}(k, x+a j)+y^{\prime}(k, x)}{a^{2}} \\
& \approx \frac{\rho(x+a h+a j)-\rho(x a j)-\rho(x+a h)+\rho(x)}{a^{2}} \cdot y^{\prime}(k, x) \\
& +\rho^{\prime}(j, x) \cdot y^{\prime \prime}(h, k, x)+\rho^{\prime}(h, x) \cdot y^{\prime \prime}(j, k, x) \\
& +\rho(x) \cdot \frac{y^{\prime}(k, x+a h+a j)-y^{\prime}(k, x+a h)-y^{\prime}(k, x+a j)+y^{\prime}(k, x)}{a^{2}} .
\end{aligned}
$$

The equivalence above is justified as follows :

$$
\begin{aligned}
\frac{\rho(x+a h+a j)-\rho(x+a j)-\rho(x+a h)+\rho(x)}{a^{2}} & \cdot\left[y^{\prime}(k, x+a h+a j)-y^{\prime}(k, x)\right] \\
= & \frac{\rho(x+a h+a j)-\rho(x+a j)-\rho(x+a h)+\rho(x)}{a} \\
& \cdot \int_{0}^{1} y^{\prime \prime}(h+j, k, x+t a(h+j)) d t \\
= & \left(\int_{0}^{1}\left[\rho^{\prime}(h, x+a j+a t h)-\rho^{\prime}(h, x+a t h)\right] d t\right) \\
& \cdot \int_{0}^{1} y^{\prime \prime}(h+j, k, x+t a(h+j)) d t
\end{aligned}
$$


$\approx 0$ since $\left|\rho^{\prime}(h, x+a j+a t h)-\rho^{\prime}(h, x+a t h)\right| \approx 0$ by 3.1. And $\frac{\rho(x+a j)-\rho(x)}{a} \approx \rho^{\prime}(j, x), \frac{\rho(x+a h)-\rho(x)}{a} \approx \rho^{\prime}(h, x)$, $\frac{y^{\prime}(k, x+a h+a j)-y^{\prime}(k, x+a j)}{a} \approx y^{\prime \prime}(h, k, x+a j)$

$\approx y^{\prime \prime}(h, k, x)$ by (3.1) and (3.2). Also it follows by (4.7) and (4.8) that

$$
\begin{aligned}
\int_{0}^{1} & \frac{F^{\prime}(h, x+a j+t a h)-F^{\prime}(h, x+t a h)}{a} d t \\
& =-\int_{0}^{1} \frac{\rho^{\prime}(k, x+a j+t a h)-\rho^{\prime}(k, x+t a h)}{a} \cdot y^{\prime}(h, x+a j+a t h) d t \\
& -\int_{0}^{1} \rho^{\prime}(k, x+t a h) \cdot \frac{y^{\prime}(h, x+a j+t a h)-y^{\prime}(h, x+t a h)}{a} d t \\
& \approx-y^{\prime}(h, x) \cdot \int_{0}^{1} \frac{\rho^{\prime}(k, x+a j+t a h)-\rho^{\prime}(k, x+t a h)}{a} d t \\
& -\rho^{\prime}(k, x) \cdot y^{\prime \prime}(j, h, x) .
\end{aligned}
$$

This equivalence can be justified by steps similar to those used before. It follows that from (4.9) we obtain

$$
\frac{\rho(x+a h+a j)-\rho(x+a h)-\rho(x+a j)+\rho(x)}{a^{2}} \cdot y^{\prime}(k, x)
$$

$$
\begin{aligned}
& +\rho^{\prime}(j, x) \cdot y^{\prime \prime}(h, k, x)+\rho^{\prime}(h, x) \cdot y^{\prime \prime}(j, k, x) \\
& +\rho(x) \cdot \frac{y^{\prime}(k, x+a h+a j)-y^{\prime}(k, x+a h)-y^{\prime}(k, x+a j)+y^{\prime}(k, x)}{a^{2}} \\
& +\rho^{\prime}(k, x) y^{\prime \prime}(j, h, x) \\
& \approx-y^{\prime}(h, x) \cdot \int_{0}^{1} \frac{\rho^{\prime}(k, x+a j+t a h)-\rho^{\prime}(k, x+t a h)}{a} d t .
\end{aligned}
$$

But the left side of the equivalence (4.10) is symmetric with respect to $h$ and $j$ and hence this holds also for the right side. We have

$$
\begin{aligned}
& y^{\prime}(h, x) \cdot \int_{0}^{1} \frac{\rho^{\prime}(k, x+a j+t a h)-\rho^{\prime}(k, x+t a h)}{a} d t \\
& \quad \approx y^{\prime}(j, x) \cdot \int_{0}^{1} \frac{\rho^{\prime}(k, x+a h+t a j)-\rho^{\prime}(k, x+t a j)}{a} d t
\end{aligned}
$$

and because of the independence of $y^{\prime} h$ and $y^{\prime} j$ follows that for all $x$ in ${ }^{*} S$ 


$$
0 \approx \int_{0}^{1} \frac{\rho^{\prime}(k, x+a h+t a j)-\rho^{\prime}(k, x+t a j)}{a} d t .
$$

Instead of differentiating we have "differenced" (4.2) according to the theory of finite differences and because of the infinitesimal value of $a$ arrive at (4.12) which is similar to the statement $\rho^{\prime \prime} h k=0$ and (4.12) would in fact imply $\rho^{\prime \prime} h k=0$ if we knew $\rho^{\prime \prime}$ existed. Thus noticing that the integral in (4.12) "ought" to be $\rho^{\prime \prime}$ we " integrate" expecting to obtain $\rho^{\prime}$. Our integration takes the following form: If $\omega$ is an infinite natural number, $x$ is in $S$, and $h$ is chosen so that $x+h$ is in $S$ then $(x+(n / \omega) h$ plays the role of $x$ and $1 / \omega$ the role of $a$ in (4.12))

$$
\begin{aligned}
0 & \approx \frac{1}{\omega} \sum_{n=0}^{\omega-1} \int_{0}^{1} \frac{\rho^{\prime}\left(k, x+\frac{n}{\omega} h+\frac{1}{\omega} h+t \frac{1}{\omega} j\right)-\rho^{\prime}\left(k, x+\frac{n}{\omega} h+t \frac{1}{\omega}\right)}{\frac{1}{\omega}} d t \\
& =\int_{0}^{1}\left(\sum_{n=0}^{\omega}\left[\rho^{\prime}\left(k, x+\frac{n}{\omega} h+\frac{1}{\omega} h+t \frac{1}{\omega} j\right)-\rho^{\prime}\left(k, x+\frac{n}{\omega} h+t \frac{1}{\omega} j\right]\right) d t\right. \\
& =\int_{0}^{1}\left[\rho^{\prime}\left(k, x+h+t \frac{1}{\omega} j\right)-\rho^{\prime}\left(k, x+t \frac{1}{\omega} j\right)\right] d t \\
& \approx \rho^{\prime}(k, x+h)-\rho^{\prime}(k, x) .
\end{aligned}
$$

The first equivalence, above, is obtained as follows: Suppose $a_{1}, \cdots, a_{\omega}$ are elements of $* E_{n}$ so that $\left|a_{i}\right| \approx 0$ for $i=1, \cdots, \omega$, where $\omega$ is an infinite natural number. Because the sequence $\left|a_{i}\right| \cdots,\left|a_{\omega}\right|$ is " finite" with respect to ${ }^{*} R$, the sequence has a maximum element, call it $\left|a_{i}\right|$. Hence

$$
\begin{aligned}
& \left|\frac{1}{\omega} \sum_{n=1}^{\omega} a_{n}\right| \leqslant \frac{1}{\omega} \sum_{n=1}^{\omega}\left|a_{n}\right| \leqslant \frac{1}{\omega}\left(\omega\left|a_{i}\right|\right) \\
& =\left|a_{i}\right| \approx 0 . \text { Thus, } \frac{1}{\omega} \sum_{n=1}^{\omega} a_{n} \approx 0 .
\end{aligned}
$$

We have shown (since $\rho^{\prime}(k, x+h)-\rho^{\prime}(k, x)$ is a number in $R$ )

$$
\rho^{\prime}(k, x+h)-\rho^{\prime}(k, x)=0
$$

for each $x$ in $S$ whenever $h$ and $k$ are orthogonal and $x+h$ belongs to $S$.

It is not difficult to show that $\rho^{\prime \prime} h k$ exists and equals $q(h \cdot k), q$ a real constant. We define

$$
R(k, h, x)=\rho^{\prime}(k, x+h)-\rho^{\prime}(k, x)-\frac{\rho^{\prime}(h, x+h)-\rho^{\prime}(h, x)}{h \cdot h}(h \cdot k) .
$$

$R$ is linear in $k$ and $R(k, h, x)=0$ when $h=k$ or $h \cdot k=0$. Thus 
$(4.14)$

$$
\rho^{\prime}(k, x+h)-\rho^{\prime}(k, x)=\frac{\rho^{\prime}(h, x+h)-\rho^{\prime}(h, x)}{h \cdot h}(h \cdot k)
$$

for all $h$ and $k$. If $j$ is orthogonal to $k$ then

$$
\begin{aligned}
& \rho^{\prime}(k, x+j+h)-\rho^{\prime}(k, x+j)=\rho^{\prime}(k, x+j+h)-\rho^{\prime}(k, x+h) \\
& \quad+p^{\prime}(k, x)-\rho^{\prime}(k, x+j)+\rho^{\prime}(k, x+h)-\rho^{\prime}(k, x) \\
& \quad=\rho^{\prime}(k, x+h)-\rho^{\prime}(k, x)
\end{aligned}
$$

by (4.13). But then by (4.14)

$$
\frac{\rho^{\prime}(h, x+j+h)-\rho^{\prime}(h, x+j)}{h \cdot h}(h \cdot k)=\frac{\rho^{\prime}(h, x+h)-\rho^{\prime}(h, x)}{h \cdot h}(h \cdot k)
$$

and thus

$$
\rho^{\prime}(h, x+j+h)-\rho^{\prime}(h, x+j)=\rho^{\prime}(h, x+h)-\rho^{\prime}(h, x) .
$$

But $j$ is not related to $h$ and so it must be the case that (referring to (4.14) again)

$$
\rho^{\prime}(k, x+h+j)-\rho^{\prime}(k, x+j)=\rho^{\prime}(k, x+h)-\rho^{\prime}(k, x)
$$

for all $h, k$ and $j$. But now

$$
\rho^{\prime}(x+k+h)-\rho(x+h)-\rho(x+k)+\rho(x)
$$

$=\rho^{\prime}(k, x+h+t k)-\rho^{\prime}(k, x+t k)$ (applying the mean-value theorem, $0<t<1)=\rho^{\prime}(k, x+h)-\rho^{\prime}(k, x)$ by $(4.15)$.

This means $\rho^{\prime}(k, x+h)-\rho^{\prime}(k, x)$ is symmetric in $h$ and $k$ and returning once more to (4.14) we have

$$
\frac{\rho^{\prime}(h, x+h)-\rho^{\prime}(h, x)}{h \cdot h}=\frac{\rho^{\prime}(k, x+k)-\rho^{\prime}(k, x)}{k \cdot k}
$$

for all $h$ and $k$, whence

$$
\frac{\rho^{\prime}(h, x+h)-\rho^{\prime}(h, x)}{h \cdot h}=q(x)
$$

which is a real function of $x$ alone. Therefore,

$$
\rho^{\prime}(k, x+h)-\rho^{\prime}(k, x)=q(x)(h \cdot k) .
$$

But also

$$
\begin{aligned}
q(x+k) & =\frac{\rho^{\prime}(h, x+k+h)-\rho^{\prime}(h, x+k)}{h \cdot h} \\
& =\frac{\rho^{\prime}(h, x+h)-\rho^{\prime}(h, x)}{h \cdot h}=q(x)
\end{aligned}
$$


and hence $q$ is a constant. Thus

$$
\rho^{\prime \prime} h k=q(h \cdot k)
$$

and we obtain

$$
\rho(x)=\alpha\left|x-x_{0}\right|^{2}+\beta
$$

for real constants $\alpha, \beta(\alpha=q / 2)$ and a constant vector $x_{0}$. But now we know that $y$ is an elementary function and our proof is complete. As stated in Nevanlinna [1], whether (4.6) holds assuming only the existence of $y^{\prime}$ remains an open question.

\section{BIBLIOGRAPHY}

1. R. Nevanlinna, Analytic functions, Princeton Mathematical Series, Number 24, Princeton, 1960.

2. - Absolute analysis, Die Grundlehren Der Mathematischen Wissenschaften, Band 102, Springer, Berlin, 1959.

3. A. Robinson, Non-standard analysis, Studies in Logic and the Foundations of Mathematics, Amsterdam, 1966.

Received March 28, 1968. The material in this paper appears in the authors doctoral dissertation at UCLA which was written under the direction of Professor Abraham Robinson.

UNIVERSITY OF SOUTH CAROLINA 



\section{PACIFIC JOURNAL OF MATHEMATICS}

\section{EDITORS}

H. ROYDEN

Stanford University

Stanford, California

\section{R. $R$ PHELPS}

University of Washington

Seattle, Washington 98105

\section{J. DugunduI}

Department of Mathematics

University of Southern California

Los Angeles, California 90007

RICHARD ARENS

University of California

Los Angeles, California 90024

\section{ASSOCIATE EDITORS}

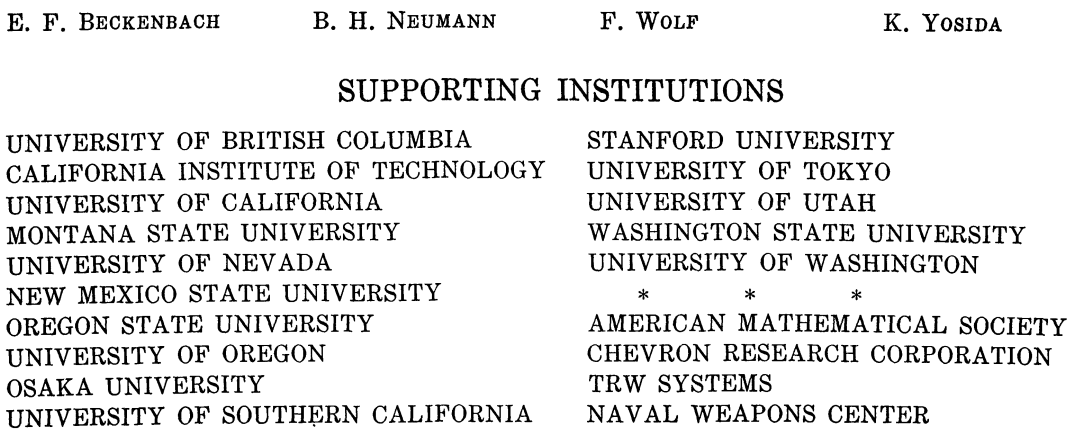

The Supporting Institutions listed above contribute to the cost of publication of this Journal, but they are not owners or publishers and have no responsibility for its content or policies.

Mathematical papers intended for publication in the Pacific Journal of Mathematics should be in typed form or offset-reproduced, double spaced with large margins. Underline Greek letters in red, German in green, and script in blue. The first paragraph or two must be capable of being used separately as a synopsis of the entire paper. It should not contain references to the bibliography. Manuscripts, in duplicate if possible, may be sent to any one of the four editors. Please classify according to the scheme of Math. Rev. 36, 1539-1546. All other communications to the editors should be addressed to the managing editor, Richard Arens, University of California, Los Angeles, California, 90024.

50 reprints are provided free for each article; additional copies may be obtained at cost in multiples of 50 .

The Pacific Journal of Mathematics is published monthly. Effective with Volume 16 the price per volume (3 numbers) is $\$ 8.00$; single issues, $\$ 3.00$. Special price for current issues to individual faculty members of supporting institutions and to individual members of the American Mathematical Society: $\$ 4.00$ per volume; single issues $\$ 1.50$. Back numbers are available.

Subscriptions, orders for back numbers, and changes of address should be sent to Pacific Journal of Mathematics, 103 Highland Boulevard, Berkeley, California, 94708.

PUBLISHED BY PACIFIC JOURNAL OF MATHEMATICS, A NON-PROFIT CORPORATION

Printed at Kokusai Bunken Insatsusha (International Academic Printing Co., Ltd.), 7-17, Fujimi 2-chome, Chiyoda-ku, Tokyo, Japan. 


\section{Pacific Journal of Mathematics}

Vol. 28, No. $2 \quad$ April, 1969

Richard Arens and Donald George Babbitt, The geometry of relativistic

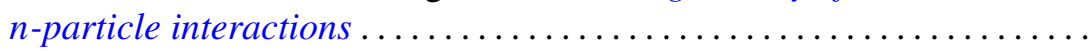

Kirby Alan Baker, Hypotopological spaces and their embeddings in lattices

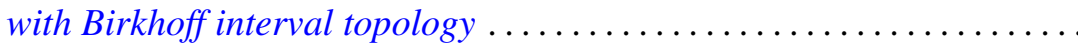

J. Lennart (John) Berggren, Finite groups in which every element is conjugate to its inverse ........................... 289

Beverly L. Brechner, Homeomorphism groups of dendrons . . . . . . . . . . . 295

Robert Ray Colby and Edgar Andrews Rutter, QF - 3 rings with zero singular ideal ................................. 303

Stephen Daniel Comer, Classes without the amalgamation property....... 309

Stephen D. Fisher, Bounded approximation by rational functions ......... 319

Robert Gaines, Continuous dependence for two-point boundary value problems..................................... 327

Bernard Russel Gelbaum, Banach algebra bundles ............... 337

Moses Glasner and Richard Emanuel Katz, Function-theoretic degeneracy criteria for Riemannian manifolds ...................... 351

Fletcher Gross, Fixed-point-free operator groups of order $8 \ldots \ldots \ldots \ldots 357$

Sav Roman Harasymiv, On approximation by dilations of distributions . . . . 363

Cheong Seng Hoo, Nilpotency class of a map and Stasheff's criterion ... . . 375

Richard Emanuel Katz, A note on extremal length and modutus.......... 381

H. L. Krall and I. M. Sheffer, Difference equations for some orthogonal polynomials ................................

Yu-Lee Lee, On the construction of lower radical properties ........... 393

Robert Phillips, Liouville's theorem........................... 397

Yum-Tong Siu, Analytic sheaf cohomology groups of dimension $n$ of

n-dimensional noncompact complex manifolds ..... . .

Michael Samuel Skaff, Vector valued Orlicz spaces. II...

James DeWitt Stein, Homomorphisms of $B^{*}$-algebras .... . .

Mark Lawrence Teply, Torsionfree injective modules .... . . .

Richard R. Tucker, The $\delta^{2}$-process and related topics. II .

David William Walkup and Roger Jean-Baptiste Robert Wets, Lifting

projections of convex polyhedra...

Thomas Paul Whaley, Large sublattices of a lattice. 